\title{
A Consumption-Investment Problem with a Diminishing Basket of Goods
}

\author{
Abdelrahim S. Mousa, Diogo Pinheiro, and Alberto A. Pinto
}

\begin{abstract}
We consider the problem faced by an economic agent trying to find the optimal strategies for the joint management of her consumption from a basket of $K$ goods that may become unavailable for consumption from some random time $\tau_{i}$ onwards, and her investment portfolio in a financial market model comprised of one risk-free security and an arbitrary number of risky securities driven by a multidimensional Brownian motion. We apply previous abstract results on stochastic optimal control problem with multiple random time horizons to obtain a sequence of dynamic programming principles and the corresponding Hamilton-Jacobi-Bellman equations. We then proceed with a numerical study of the value function and corresponding optimal strategies for the problem under consideration in the case of discounted constant relative risk aversion utility functions (CRRA).
\end{abstract}

\section{Introduction}

The origin of Optimal Control is related with Calculus of Variations, which started to be developed to deal with problems arising in Physics from the middle of $1600 \mathrm{~s}$ onwards. One of the main techniques used to address optimal control problems is the dynamic programming principle, introduced by Bellman [1-3] in 1950s. The dynamic programming principle has been extended to address stochastic optimal control problems $[4,14,15,18]$, providing a backwards recursive relation for the value function associated with such problems and, under additional conditions, a nonlinear partial differential equation known as the Hamilton-Jacobi-Bellman

\footnotetext{
A.S. Mousa $(\square)$

Faculty of Science, Department of Mathematics, Birzeit University, Ramallah, Palestine e-mail: asaid@birzeit.edu

D. Pinheiro

Department of Mathematics, Brooklyn College of the City University of New York, NY, USA e-mail: dpinheiro@brooklyn.cuny.edu

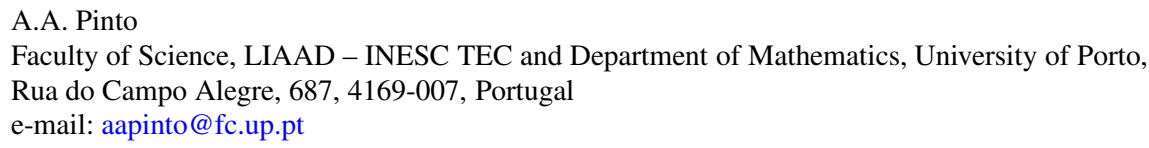


(HJB) equation. For further details on this subject we refer to the textbooks by Fleming and Rishel [12], Fleming and Soner [13] and Yong and Zhou [21]. Another key technique to address optimal control problems is the Pontryagin's maximum principle [9, 10]. For further details on this approach see Haussmann [16], Bismut [5-7] and Yong and Zhou [21].

More recently there has been considerable interest in the study of optimization problems with an objective functional and state variable dynamics depending on a random time horizon. Such problems were often considered in the context of mathematical finance. See, for instance, the papers [8, 11, 20] for further details.

A general framework for this class of problems is considered in [19]. In that paper a family of stochastic optimal control problems is considered with the property that the objective functional depends on multiple random time horizons. The state variable follows a stochastic differential equation driven by a standard multi-dimensional Brownian motion. For that class of stochastic optimal control problems, a sequence of dynamic programming principles and the corresponding Hamilton-Jacobi-Bellman equations are derived. In the current paper we discuss an application of the abstract results of [19] to a consumption-investment problem with a diminishing basket of goods.

We consider an economic agent investing in a financial market model consisting of one risk-free security and an arbitrary number of risky securities driven by a multi-dimensional Brownian motion. We assume that the economic agent is consuming from a basket of $K$ goods that may become unavailable for consumption from some random time $\tau_{i}$ onwards. The economic agent goal is to jointly maximize: (i) the utility derived from consumption of the goods available at each instant of time; and (ii) the utility derived from wealth at some multiple random instants of time, representing either the moment at which a given good becomes unavailable or the deterministic horizon $T$.

This paper is organized as follows. In Sect. 2 we review the main results in [19], i.e. the sequence of dynamic programming principles and corresponding HamiltonJacobi-Bellman equations (HJB). In Sect. 3 we set up the optimization problem under consideration here by introducing the underlying financial market and the corresponding wealth process. We restate the stochastic optimal control problem under consideration as one with a fixed planning horizon, and derive a sequence of dynamic programming principles and the corresponding Hamilton-Jacobi-Bellman equations. We use the results discussed in Sect. 2 to proceed with detailed analysis of our consumption problem with multiple random time horizons. We conclude in Sect. 4.

\section{Preliminary Results}

In this section we review the problem formulation of [19]. Let $K \in \mathbb{N}$ be fixed and $T>0$ be a finite time horizon. Assume that $(\Omega, \mathscr{F}, \mathbb{P})$ is a complete probability space equipped with a filtration $\mathbb{F}=\left\{\mathscr{F}_{t}, t \in[0, T]\right\}$ given by the $\mathbb{P}$-augmentation 
of the filtration generated by the $M$-dimensional Brownian motion $W(t), \sigma\{W(s)$, $s \leq t\}$ for $t \geq 0$.

We denote by $L_{\mathscr{F}_{t}}^{1}([0, T] ; \mathbb{R})$ the set of all $\left\{\mathscr{F}_{t}\right\}_{t \geq 0}$-adapted $\mathbb{R}$-valued processes $x(\cdot)$ such that

$$
E\left[\int_{0}^{T}|x(t)| \mathrm{d} t\right]<\infty
$$

and by $L_{\mathscr{F}_{T}}^{1}(\Omega ; \mathbb{R})$ the set of $\mathbb{R}$-valued $\mathscr{F}_{T}$-measurable random variables $X$ such that $E[|X|]$ is finite. The spaces $L_{\mathscr{F}_{t}}^{1}([0, T] ; \mathbb{R})$ and $L_{\mathscr{F}_{T}}^{1}(\Omega ; \mathbb{R})$ are defined on the filtered probability space $(\Omega, \mathscr{F}, \mathbb{F}, \mathbb{P})$.

Assume that $\tau_{1}, \tau_{2}, \ldots, \tau_{K}$ are non-negative independent and identically distributed continuous random variables defined on the probability space $(\Omega, \mathscr{F}, \mathbb{P})$, with distribution function $F:[0, \infty) \rightarrow[0,1]$ and density $f:[0, \infty) \rightarrow \mathbb{R}^{+}$. Moreover, assume that the random variables $\tau_{i}$ are completely independent of the filtration $\mathbb{F}$ for all $i \in\{1,2, \ldots, K\}$.

Let $\tau_{(1)} \leq \tau_{(2)} \leq \ldots \leq \tau_{(K)}$ be the order statistics associated with the random variables $\tau_{1}, \tau_{2}, \ldots, \tau_{K}$. Define the following finite sequence of times:

(i) the sequence $\left(\xi_{i}\right)_{i=1}^{K}$ is given by

$$
\xi_{i}=\min \left\{\tau_{i}, T\right\}, \quad i=1,2, \ldots, K
$$

(ii) the sequence $\left(t_{i}\right)_{i=0}^{K}$ is given by $t_{0}=0$ and

$$
t_{i}=\min \left\{\tau_{(i)}, T\right\}, \quad i=1,2, \ldots, K .
$$

Using the sequence $\left(t_{i}\right)$, we introduce the sequence of random intervals $\Delta_{k}$ as being given by

$$
\Delta_{k}=\left[t_{k-1}, t_{k}\right), \quad k \in\{1,2, \ldots, K\} .
$$

For each $t \in\left[0, t_{K}\right)$, let $I(t)$ be the set of indices given by

$$
I(t)=\left\{i \in\{1,2, \ldots, K\}: \tau_{i}>t\right\}
$$

and let $\kappa:\left[0, t_{K}\right) \rightarrow\{1,2, \ldots, K\}$ be the function assigning to each $t \in\left[0, t_{K}\right)$ the cardinality of the set $I(t)$.

We now introduce some technical assumptions:

(A1) $\quad(U, d)$ is a Polish space.

(A2) The maps $f_{k}:[0, T] \times \mathbb{R}^{N} \times U \rightarrow \mathbb{R}^{N}, g_{k}:[0, T] \times \mathbb{R}^{N} \times U \rightarrow \mathbb{R}^{N \times M}$, $\Psi_{k}:[0, T] \times \mathbb{R}^{N} \rightarrow \mathbb{R}$ and $L_{k}:[0, T] \times \mathbb{R}^{N} \times U \rightarrow \mathbb{R}, k=1,2, \ldots, K$, are uniformly continuous with respect to all its variables, Lipschitz continuous with respect to the variable $x$, and bounded when restricted to $x=0$. 
Let $f:\left[0, t_{K}\right] \times \mathbb{R}^{N} \times U \rightarrow \mathbb{R}^{N}$ and $g:\left[0, t_{K}\right] \times \mathbb{R}^{N} \times U \rightarrow \mathbb{R}^{N \times M}$ be the functions defined through the relations

$$
f(t, x, u)=f_{k}(t, x, u) \quad \text { and } \quad g(t, x, u)=g_{k}(t, x, u)
$$

for every $(t, x, u) \in \Delta_{k} \times \mathbb{R}^{N} \times U$, extended by continuity to $t=t_{K}$. Consider the stochastic controlled system

$$
\begin{aligned}
\mathrm{d} x(t) & =f(t, x(t), u(t)) \mathrm{d} t+g(t, x(t), u(t)) \mathrm{d} W(t), \quad t \in\left[0, t_{K}\right], \\
x(0) & =x_{0}
\end{aligned}
$$

and the objective functional

$$
J(u(\cdot))=E\left[\sum_{i=1}^{K}\left(\int_{0}^{\xi_{i}} L_{i}(t, x(t), u(t)) \mathrm{d} t+\Psi_{i}\left(\xi_{i}, x\left(\xi_{i}\right)\right)\right)\right],
$$

where $x(t)$ is a state trajectory in $\mathbb{R}^{N}$ with the corresponding control $u(t) \in U$, $L_{i}(\cdot, x(\cdot), u(\cdot)) \in L_{\mathscr{F}_{t}}^{1}\left(\left[0, t_{K}\right] ; \mathbb{R}\right)$ and $\Psi_{i}\left(\xi_{i}, x\left(\xi_{i}\right)\right) \in L_{\mathscr{F}_{\xi_{i}}}^{1}(\Omega ; \mathbb{R}), i=1,2, \ldots, K$.

To use the dynamic programming methods, for any initial condition $(s, y) \in$ $\left[0, t_{K}\right) \times \mathbb{R}^{N}$, we consider the state equation:

$$
\begin{aligned}
\mathrm{d} x(t) & =f(t, x(t), u(t)) \mathrm{d} t+g(t, x(t), u(t)) \mathrm{d} W(t), \quad t \in\left[s, t_{K}\right], \\
x(s) & =y,
\end{aligned}
$$

along with the objective functional

$$
J(s, y ; u(\cdot))=E\left[\sum_{i \in I(s)} \int_{s}^{\xi_{i}} L_{i}(t, x(t), u(t)) \mathrm{d} t+\Psi_{i}\left(\xi_{i}, x\left(\xi_{i}\right)\right)\right]
$$

For each $s \in\left[0, t_{K}\right)$, we denote by $\mathscr{U}^{w}[s, T]$ the set of all 5 -tuples $(\Omega, \mathscr{F}, \mathbb{P}, W(\cdot), u(\cdot))$ for which the following conditions hold:

(i) $(\Omega, \mathscr{F}, \mathbb{P})$ is a complete probability space;

(ii) $\{W(t)\}_{t \geq s}$ is an $M$-dimensional standard Brownian motion defined on $(\Omega, \mathscr{F}, \mathbb{P})$ over $[s, T]$, and $\mathscr{F}_{t}^{s}$ is the filtration generated by $\{W(t)\}_{t \geq s}$, $\sigma\{W(r): s \leq r \leq t\}$, augmented by all the $\mathbb{P}$-null sets in $\mathscr{F}$;

(iii) $u:\left[s, t_{K}\right] \times \Omega \rightarrow U$ is an $\left\{\mathscr{F}_{t}^{s}\right\}_{t \geq s}$-adapted process on $(\Omega, \mathscr{F}, \mathbb{P})$;

(iv) under $u(\cdot)$, for any $y \in \mathbb{R}^{N}$ Eq. (1) admits a unique solution $x(\cdot)$ on $\left(\Omega, \mathscr{F},\left\{\mathscr{F}_{t}^{s}\right\}_{t \geq s}, \mathbb{P}\right)$.

We call $\mathscr{U}^{w}[s, T]$ the set of weak admissible controls. 
Using the notations introduced above, the goal is to find a weak admissible control $u(\cdot) \in \mathscr{U}^{w}[s, T]$ such that the following identity holds

$$
V(s, y)=\sup _{u(\cdot) \in \mathscr{U}^{w}[s, T]} J(s, y ; u(\cdot)), \quad(s, y) \in\left[0, t_{K}\right) \times \mathbb{R}^{N} .
$$

The function $V(s, y)$ defined above is referred to as the value function.

For every $0 \leq s \leq t$ and every $i, k \in\{1,2, \ldots, K\}$ such that $i \geq k$, let $G_{\tau_{(i)} \mid \tau_{(k)}}^{ \pm}(t, s)$ denote the conditional probabilities

$$
\begin{aligned}
& G_{\tau_{(i)} \mid \tau_{(k)}}^{+}(t, s)=P\left(\left\{\tau_{(i)}>t\right\} \mid\left\{\tau_{(k)}>s\right\}\right) \\
& G_{\tau_{(i)} \mid \tau_{(k)}}^{-}(t, s)=P\left(\left\{\tau_{(i)} \leq t\right\} \mid\left\{\tau_{(k)}>s\right\}\right)
\end{aligned}
$$

and let $g_{\tau_{(i)} \mid \tau_{(k)}}^{-}(t, s)$ denote the density function of $G_{\tau_{(i)} \mid \tau_{(k)}}^{-}(t, s)$, given by

$$
g_{\tau_{(i)} \mid \tau_{(k)}}^{-}(t, s)=\frac{\mathrm{d}}{\mathrm{d} t} G_{\tau_{(i)} \mid \tau_{(k)}}^{-}(t, s) .
$$

Furthermore, for every $k \in\{1,2, \ldots, K\}$ such that $t_{k-1}<T, i \in\{1,2, \ldots, K\}$ such that $i \geq k, j \in I\left(t_{k-1}\right)$ and $t_{k-1} \leq s \leq t$, define the conditional Lagrangian function to be

$$
\mathscr{L}_{\tau_{(i)} \mid \tau_{(k)}}^{j}(t, s, x, u)=G_{\tau_{(i)} \mid \tau_{(k)}}^{+}(t, s) L_{j}(t, x, u)+g_{\tau_{(i)} \mid \tau_{(k)}}^{-}(t, s) \Psi_{j}(t, x) .
$$

We now state the two main results of [19].

Theorem 1 (Dynamic programming principle) Assume that conditions (A1) and (A2) hold. Then, for every $k \in\{1,2, \ldots, K\}$ such that $t_{k-1}<T$, the restriction of the value function $V$ to the set $\Delta_{k} \times \mathbb{R}^{N}$ is identically equal to the function determined by the recursive relation

$$
\begin{aligned}
& V(s, y)=\sup _{u(\cdot) \in \mathscr{U}^{w}[s, T]} E\left[G_{\tau_{(k)} \mid \tau_{(k)}}^{+}(\hat{s}, s) V(\hat{s}, x(\hat{s} ; s, y, u(\cdot)))\right. \\
& \left.+\frac{1}{\kappa\left(t_{k-1}\right)} \sum_{i=k}^{K} \sum_{j \in I\left(t_{k-1}\right)} \int_{s}^{\hat{s}} \mathscr{L}_{\tau_{(i)} \mid \tau_{(k)}}^{j}(t, s, x(t ; s, y, u(\cdot)), u(t)) \mathrm{d} t \mid s \in \Delta_{k}\right], \hat{s} \in[s, T]
\end{aligned}
$$

combined with the boundary condition

$$
V(T, x)=\sum_{j \in I\left(t_{k-1}\right)} \Psi_{j}(T, x) .
$$


Let $I \subseteq \mathbb{R}$ be an interval and denote by $C^{1,2}\left(I \times \mathbb{R}^{N} ; \mathbb{R}\right)$ the set of all continuous functions $V: I \times \mathbb{R}^{N} \rightarrow \mathbb{R}$ such that $V_{t}, V_{x}$, and $V_{x x}$ are all continuous functions of $(t, x)$. Using the sequence of Dynamic programming principles from the previous theorem, we were able to derive the associated sequence of Hamilton-JacobiBelman equations.

Theorem 2 Suppose that conditions (A1) and (A2) hold. Additionally, assume that for every $i, j \in\{1,2, \ldots, K\}$ such that $i \geq j$ the conditional density functions $g_{\tau_{(i)} \mid \tau_{(j)}}^{-}(t, s)$ are uniformly continuous with respect to $t$.

Let $k \in\{1,2, \ldots, K\}$ be such that $t_{k-1}<T$ and assume that the value function $V$ is such that $V \in C^{1,2}\left(\Delta_{k} \times \mathbb{R}^{N} ; \mathbb{R}\right)$. Then, on each set $\Delta_{k} \times \mathbb{R}^{N}$ the value function $V$ is identically equal to the solution of the Hamilton-Jacobi-Bellman equation

$$
\begin{cases}V_{t}-g_{\tau_{(k)} \mid \tau_{(k)}}^{-}(t, t) V+\sup _{u \in U} \mathscr{H}^{k}\left(t, x, u, V_{x}, V_{x x}\right)=0 & \\ V(T, x)=\sum_{j \in I\left(t_{k-1}\right)} \Psi_{j}(T, x), & (t, x) \in\left[t_{k-1}, T\right) \times \mathbb{R}^{N},\end{cases}
$$

where the Hamiltonian function $\mathscr{H}^{k}$ is given by

$$
\begin{aligned}
\mathscr{H}^{k}(t, x, u, p, B)= & \sum_{j \in I\left(t_{k-1}\right)}\left(L_{j}(t, x, u)+G_{k}(t) \Psi_{j}(t, x)\right) \\
& +\left\langle p, f_{k}(t, x, u)\right\rangle+\frac{1}{2} \operatorname{tr}\left(g_{k}^{T}(t, x, u) B g_{k}(t, x, u)\right)
\end{aligned}
$$

for all $(t, x, u, p, B) \in[0, T] \times \mathbb{R}^{N} \times U \times \mathbb{R}^{N} \times \mathscr{S}^{N}, \mathscr{S}^{N}$ is the set of all $N \times N$ symmetric real matrices and $G_{k}(t)$ is given by

$$
G_{k}(t)=\frac{1}{\kappa\left(t_{k-1}\right)} \sum_{i=k}^{K} g_{\tau_{(i)} \mid \tau_{(k)}}^{-}(t, t) .
$$

\section{An Application to a Consumption-Investment Decision Problem}

Consider a financial market consisting of one risk-free security and a fixed number $N \geq 1$ of risky securities. The asset prices $\left(S_{0}(t)\right)_{0 \leq t \leq T}$ and $\left(S_{n}(t)\right)_{0 \leq t \leq T}, n=$ $1, \ldots, N$, evolve according to the differential equations:

$$
\begin{aligned}
\mathrm{d} S_{0}(t) & =r(t) S_{0}(t) \mathrm{d} t, & S_{0}(0) & =s_{0}>0, \\
\mathrm{~d} S_{n}(t) & =\mu_{n}(t) S_{n}(t) \mathrm{d} t+S_{n}(t) \sum_{m=1}^{M} \sigma_{n m}(t) \mathrm{d} W_{m}(t), & S_{n}(0) & =s_{n}>0,
\end{aligned}
$$


where $W(t)=\left(W_{1}(t), \ldots, W_{M}(t)\right)^{T}$ is a standard $M$-dimensional Brownian motion on a filtered complete probability space $(\Omega, \mathscr{F}, \mathbb{F}, \mathbb{P}), r(t)$ is the riskless interest rate, $\mu(t)=\left(\mu_{1}(t), \ldots, \mu_{N}(t)\right) \in \mathbb{R}^{N}$ is the vector of the risky-assets appreciation rates and $\sigma(t)=\left(\sigma_{n m}(t)\right)_{1 \leq n \leq N, 1 \leq m \leq M}$ is the matrix of risky-assets volatilities. Each sub- $\sigma$-algebra $\mathscr{F}_{t}$ represents the information available to any financial market observer during the time span $[0, t]$.

For simplicity of exposition, we assume that the coefficients $r(t), \mu(t)$ and $\sigma(t)$ are deterministic continuous functions on the interval $[0, T]$. We also assume that the interest rate $r(t)$ is positive for all $t \in[0, T]$ and the matrix $\sigma(t)$ is such that $\sigma \sigma^{T}$ is non-singular for Lebesgue almost all $t \in[0, T]$ and satisfies the following integrability condition

$$
\sum_{n=1}^{N} \sum_{m=1}^{M} \int_{0}^{T} \sigma_{n m}^{2}(t) \mathrm{d} t<\infty
$$

Furthermore, we suppose that there exists an $\left(\mathscr{F}_{t}\right)_{0 \leq t \leq T}$-progressively measurable process $\pi(t) \in \mathbb{R}^{M}$, called the market price of risk, such that for Lebesgue-almostevery $t \in[0, T]$ the risk premium

$$
\alpha(t)=\left(\mu_{1}(t)-r(t), \ldots, \mu_{N}(t)-r(t)\right) \in \mathbb{R}^{N}
$$

is related to $\pi(t)$ by the equation

$$
\alpha^{T}(t)=\sigma(t) \pi^{T}(t) \quad \text { a.s. }
$$

and the following two conditions hold

$$
\begin{aligned}
& \int_{0}^{T}\|\pi(t)\|_{M}^{2}<\infty \quad \text { a.s. } \\
& E\left[\exp \left(-\int_{0}^{T} \pi(s) \mathrm{d} W(s)-\frac{1}{2} \int_{0}^{T}\|\pi(s)\|_{M}^{2} \mathrm{~d} s\right)\right]=1,
\end{aligned}
$$

where $\|\cdot\|_{M}$ denotes the Euclidean norm in $\mathbb{R}^{M}$. The existence of $\pi(t)$ ensures the absence of arbitrage opportunities in the financial market defined above. See [17] for further details on market viability.

Let $\tau_{1}, \tau_{2}, \ldots, \tau_{K}$ be non-negative independent and identically distributed continuous random variables defined on the probability space $(\Omega, \mathscr{F}, \mathbb{P})$. Moreover, assume that $\tau_{1}, \tau_{2}, \ldots, \tau_{K}$ are independent of the filtration $\mathbb{F}$ generated by the Brownian motion $W(t)$. Let $\left(\xi_{i}\right)$ and $\left(t_{i}\right)$ be as defined in Sect. 2.

We will consider the problem faced by an economic agent investing in the financial market described above and consuming from a basket of $K$ goods that may 
become unavailable for consumption from some time $\tau_{i}$ onwards. The economic agent goal is to jointly maximize:

(i) the utility derived from consumption of the goods available at each instant of time;

(ii) the utility derived from wealth at the multiple random instants of time $\xi_{1}, \xi_{2}, \ldots, \xi_{K}$, representing either the moment at which a given good becomes unavailable or the deterministic horizon $T$.

For each $i=1,2, \ldots, K$ and $t \in\left[0, t_{K}\right)$, let $c_{i}(t)$ denote the economic agent consumption of the $i$ th good at the instant of time $t$. The consumption process is given by

$$
c(t)=\left(c_{1}(t), \ldots, c_{K}(t)\right) .
$$

We assume that the consumption process is a $\left(\mathscr{F}_{t}\right)$-progressively measurable nonnegative process with the property that each component $c_{i}(\cdot)$ is non-negative and satisfies the following integrability condition

$$
\int_{0}^{\xi_{i}}\|c(t)\|_{K}^{2} \mathrm{~d} t<\infty \quad \text { a.s. . }
$$

For each $n=0,1, \ldots, N$ and $t \in\left[0, t_{K}\right]$, let $\theta_{n}(t)$ denote the fraction of the economic agent wealth allocated to the asset $S_{n}$ at time $t$. The portfolio process is given by

$$
\Theta(t)=\left(\theta_{0}(t), \theta_{1}(t), \cdots, \theta_{N}(t)\right) \in \mathbb{R}^{N+1},
$$

where

$$
\sum_{n=0}^{N} \theta_{n}(t)=1, \quad 0 \leq t \leq t_{K} .
$$

We assume that the portfolio process is $\left(\mathscr{F}_{t}\right)$-progressively measurable and that

$$
\int_{0}^{t_{K}}\|\Theta(t)\|_{N+1}^{2} \mathrm{~d} t<\infty \quad \text { a.s. . }
$$

Using relation (2), we can always write $\theta_{0}(t)$ in terms of $\theta_{1}(t), \ldots, \theta_{N}(t)$. From now on, we will define the portfolio process in terms of the reduced portfolio process $\theta(t) \in \mathbb{R}^{N}$ given by

$$
\theta(t)=\left(\theta_{1}(t), \theta_{2}(t), \cdots, \theta_{N}(t)\right) \in \mathbb{R}^{N} .
$$


We define the wealth process $X(t)$, for $t \in\left[0, t_{K}\right]$ by

$$
X(t)=x-\sum_{i=1}^{K} \int_{0}^{\xi_{i} \wedge t} c_{i}(s) \mathrm{d} s+\sum_{n=0}^{N} \int_{0}^{t} \frac{\theta_{n}(s) X(s)}{S_{n}(s)} \mathrm{d} S_{n}(s)
$$

where $x$ is the economic agent initial wealth. The last equation can be rewritten in the differential form

$$
\begin{aligned}
\mathrm{d} X(t)= & \left(-\sum_{i \in I(t)} c_{i}(t)+\left(\theta_{0}(t) r(t)+\sum_{n=1}^{N} \theta_{n}(t) \mu_{n}(t)\right) X(t)\right) \mathrm{d} t \\
& +\sum_{n=1}^{N} \theta_{n}(t) X(t) \sum_{m=1}^{M} \sigma_{n m}(t) \mathrm{d} W_{m}(t),
\end{aligned}
$$

where $I(t)$ is the index set defined in Sect. 2.

Let us denote by $\mathscr{A}(x)$ the set of all admissible decision strategies, i.e. all admissible choices for the control variables $(c, \theta) \in \mathbb{R}^{K+N}$. The dependence of $\mathscr{A}(x)$ on $x$ denotes the restriction imposed on the wealth process by the boundary condition $X(0)=x$. Similarly, let us denote by $\mathscr{A}(t, x)$ the set of all admissible decision strategies $(c, \theta)$ for the dynamics of the wealth process with boundary condition $X(t)=x$.

The economic agent problem is to find a consumption-investment strategy $(c, \theta) \in \mathscr{A}(x)$ which maximizes the expected utility

$$
V(x)=\sup _{(c, \theta) \in \mathscr{A}(x)} E\left[\sum_{i=1}^{K} \int_{0}^{\xi_{i}} L_{i}\left(t, c_{i}(t)\right) \mathrm{d} t+\Psi_{i}\left(\xi_{i}, X\left(\xi_{i}\right)\right)\right]
$$

where $L_{i}\left(t, c_{i}(t)\right), i=1,2, \ldots, K$, is the utility functions describing the economic agent preferences regarding the $i$ th good consumption over the random time interval $\left[0, \xi_{i}\right]$, and $\Psi_{i}\left(\xi_{i}, X\left(\xi_{i}\right)\right), i=1,2, \ldots, K$, are the utility functions describing the economic agent preferences concerning the amount of wealth held at the instants of time $\xi_{1}, \xi_{2}, \ldots, \xi_{K}$.

Assume that the conditions of Theorem 2 hold. Then, for every $k \in\{1,2, \ldots, K\}$ such that $t_{k-1}<T$ the value function $V$ restricted to the set $\Delta_{k} \times \mathbb{R}^{N}$ is identically equal to the solution of the Hamilton-Jacobi-Bellman equation:

$$
\left\{\begin{array}{l}
V_{t}-g_{\tau_{(k)} \mid \tau_{(k)}}^{-}(t, t) V+\sup _{(c, \theta) \in \mathscr{A}(t, x)} \mathscr{H}^{k}\left(t, x, c, \theta, V_{x}, V_{x x}\right)=0 \\
V(T, x)=\sum_{i \in I\left(t_{k-1}\right)} \Psi_{i}(T, x), \quad(t, x) \in\left[t_{k-1}, T\right) \times \mathbb{R}^{N},
\end{array}\right.
$$


where the Hamiltonian function $\mathscr{H}^{k}$ is given by

$$
\begin{aligned}
\mathscr{H}^{k}(t, x, c, \theta, p, B)= & \sum_{i \in I\left(t_{k}-1\right)}\left(L_{i}\left(t, c_{i}\right)+G_{k}(t) \Psi_{i}(t, x)\right) \\
& +\left(-\sum_{i \in I\left(t_{k-1}\right)} c_{i}(t)+\left(r(t)+\sum_{n=1}^{N} \theta_{n}\left(\mu_{n}(t)-r(t)\right)\right) x\right) p \\
& +\frac{x^{2}}{2} \sum_{m=1}^{M}\left(\sum_{n=1}^{N} \theta_{n} \sigma_{n m}(t)\right)^{2} B
\end{aligned}
$$

and $G_{k}(t)$ is as given in the statement of Theorem 2.

\subsection{Constant Relative Risk Aversion Utility Functions}

We will now focus our attention on the class of discounted CRRA utility functions, given by

$$
L_{i}\left(t, c_{i}\right)=\mathrm{e}^{-\rho t} \frac{c_{i}^{\gamma_{i}}}{\gamma_{i}}, \quad \Psi_{i}(t, x)=\mathrm{e}^{-\rho t} \frac{x^{\beta_{i}}}{\beta_{i}}, \quad i=1,2, \ldots, K,
$$

where the risk aversion parameters $\gamma_{i}$ and $\beta_{i}$ are different from zero and strictly less than one for every $i \in\{1,2, \ldots, K\}$, and the discount rate $\rho$ is positive.

Computing the first-order conditions for a regular interior maximum of $\mathscr{H}^{k}$ with respect to $(c, \theta)$, we obtain the following two equalities:

$$
\begin{aligned}
-V_{x}(t, x)+\frac{\mathrm{d} L_{i}}{\mathrm{~d} c_{i}}\left(t, c_{i}^{*}\right) & =0, \quad i \in I\left(t_{k-1}\right) \\
x V_{x}(t, x) \alpha+x^{2} V_{x x}(t, x) \theta^{*} \sigma \sigma^{T} & =0_{\mathbb{R}^{N}} .
\end{aligned}
$$

Thus, solving with respect to $c$ and $\theta$, we are able to express the optimal strategies in terms of the value function derivatives as

$$
\begin{aligned}
c_{i}^{*}(t, x) & =\left(e^{\rho t} V_{x}(t, x)\right)^{-1 /\left(1-\gamma_{i}\right)}, \quad i \in I\left(t_{k-1}\right) \\
\theta^{*}(t) & =-\frac{V_{x}(t, x)}{x V_{x x}(t, x)} \alpha(t) \xi
\end{aligned}
$$

where $\xi$ denotes the non-singular square matrix $\left(\sigma \sigma^{T}\right)^{-1}$. Using the utility functions (4) and substituting $c_{i}^{*}$ and $\theta^{*}$ in the HJB equation (3), we arrive at the following 
partial differential equation

$$
\begin{aligned}
& V_{t}(t, x)-g_{\tau_{(k)} \mid \tau_{(k)}}^{-}(t, t) V(t, x)+r(t) x V_{x}(t, x) \\
& -\Sigma(t) \frac{\left(V_{x}(t, x)\right)^{2}}{V_{x x}(t, x)}+G_{k}(t) \mathrm{e}^{-\rho t} \sum_{i \in I\left(t_{k-1}\right)} \frac{x^{\beta_{i}}}{\beta_{i}} \\
& +\sum_{i \in I\left(t_{k-1}\right)}\left(\frac{1-\gamma_{i}}{\gamma_{i}}\right) e^{-\rho t /\left(1-\gamma_{i}\right)}\left(V_{x}(t, x)\right)^{-\gamma_{i} /\left(1-\gamma_{i}\right)}=0,
\end{aligned}
$$

where $\Sigma(t)$ is given by

$$
\Sigma(t)=\alpha(t) \xi \alpha^{T}(t)-\frac{1}{2}\left\|\sigma^{T}(t) \xi \alpha^{T}(t)\right\|_{M}^{2}
$$

and the terminal condition is given by

$$
V(T, x)=\sum_{i \in I\left(t_{k-1}\right)} \Psi_{i}(T, x) .
$$

The following result provides the optimal strategies for discounted CRRA utility functions in case where the risk aversion parameters are all equal. Quite naturally, we obtain that the optimal strategy consists in consuming identical amounts of each good available for consumption at a given time.

Corollary 1 Let $\xi$ denote the non-singular square matrix given by $\left(\sigma \sigma^{T}\right)^{-1}$. Assume that the risk aversion parameters $\gamma_{i}$ and $\beta_{i}$ are all equal. Then, for every $k \in\{1,2, \ldots, K\}$ such that $t_{k-1}<T$ the value function $V$ restricted to the set $\Delta_{k} \times \mathbb{R}$ is of the form

$$
V(t, x)=\kappa\left(t_{k-1}\right) a_{k}(t) \frac{x^{\gamma}}{\gamma},
$$

where $a_{k}(t)$ is the solution of the boundary value problem

$$
\begin{aligned}
& \frac{d a_{k}(t)}{d t}+\Phi_{k}(t) a_{k}(t)+\eta(t)\left(\kappa\left(t_{k-1}\right) a_{k}(t)\right)^{-\gamma /(1-\gamma)}+G_{k}(t) \mathrm{e}^{-\rho t}=0 \\
& a_{k}(T)=e^{-\rho T},
\end{aligned}
$$

the function $\Sigma(t)$ is as given in (7), $G_{k}(t)$ is as given in the statement of Theorem 2 and $\Phi_{k}(t)$ and $\eta(t)$ are given by

$$
\begin{aligned}
\Phi_{k}(t) & =\gamma\left(r(t)+\frac{\Sigma(t)}{1-\gamma}\right)-g_{\tau_{(k)} \mid \tau_{(k)}}^{-}(t, t), \\
\eta(t) & =\gamma(1-\gamma) e^{-\rho t /(1-\gamma)} .
\end{aligned}
$$


Furthermore, on each set $\Delta_{k} \times \mathbb{R}$, the optimal strategies are given by

$$
\begin{aligned}
& c_{i}^{*}(t, x)=x\left(\kappa\left(t_{k-1}\right) e^{\rho t} a_{k}(t)\right)^{-1 /(1-\gamma)}, \quad i \in I\left(t_{k-1}\right) \\
& \theta^{*}(t, x)=\frac{1}{1-\gamma} \alpha(t) \xi
\end{aligned}
$$

Proof Start by noting that in the case where the risk aversion parameters $\gamma_{i}$ and $\beta_{i}$ are all equal (we denote such common value by $\gamma$ ), the partial differential equation (6) becomes

$$
\begin{aligned}
& V_{t}(t, x)-g_{\tau_{(k)} \mid \tau_{(k)}}^{-}(t, t) V(t, x)+r(t) x V_{x}(t, x)-\Sigma(t) \frac{\left(V_{x}(t, x)\right)^{2}}{V_{x x}(t, x)} \\
& +\sum_{i \in I\left(t_{k-1}\right)} \frac{1}{\gamma}\left(G_{k}(t) \mathrm{e}^{-\rho t} x^{\lambda}+(1-\gamma) e^{-\rho t /(1-\gamma)}\left(V_{x}(t, x)\right)^{-\gamma /(1-\gamma)}\right)=0,
\end{aligned}
$$

where $\Sigma(t)$ is as given in (7), $G_{k}(t)$ is as given in the statement of Theorem 2 and the terminal condition is as given in (8).

We consider an ansatz of the form

$$
V(t, x)=\sum_{i \in I\left(t_{k-1}\right)} a_{k}(t) \frac{x^{\gamma}}{\gamma}=\kappa\left(t_{k-1}\right) a_{k}(t) \frac{x^{\gamma}}{\gamma}
$$

and substitute it in (9). A simple computation implies that $a_{k}(t)$ is determined by the following boundary value problem

$$
\begin{aligned}
& \frac{\mathrm{d} a_{k}(t)}{\mathrm{d} t}+\Phi_{k}(t) a_{k}(t)+\eta(t)\left(\kappa\left(t_{k-1}\right) a_{k}(t)\right)^{-\gamma /(1-\gamma)}+G_{k}(t) \mathrm{e}^{-\rho t}=0 \\
& a_{k}(T)=e^{-\rho T},
\end{aligned}
$$

where $\Phi_{k}(t)$ and $\eta(t)$ are as given in the statement of this corollary. Furthermore, using (5) we obtain that optimal strategies on $\Delta_{k} \times \mathbb{R}$ are given by

$$
\begin{aligned}
c_{i}^{*}(t, x) & =x\left(\kappa\left(t_{k-1}\right) e^{\rho t} a_{k}(t)\right)^{-1 /(1-\gamma)}, \quad i \in I\left(t_{k-1}\right) \\
\theta^{*}(t, x) & =\frac{1}{1-\gamma} \alpha(t) \xi
\end{aligned}
$$

concluding the proof. 


\subsection{Numerical Solution}

Before concluding, we provide a numerical example where the risk aversion parameters do not coincide. We take the deterministic fixed horizon to be $T=20$ and consider a financial market with one risk-free security and one risky asset $(N=1)$ driven by a one-dimensional Brownian motion $(M=1)$. The riskless interest rate is $r=0.04$, the risky asset appreciation rate is $\mu=0.08$ and its volatility is $\sigma=0.19$, whereas the discount rate is taken to be $\rho=0.04$. We assume that there is a basket of three goods available for consumption $(K=3)$ up until some random times $\tau_{1}, \tau_{2}, \tau_{3}$, which are assumed to be independent and identically distributed non-negative random variables with distribution function given by

$$
F(t)=1-\exp \left(-0.0010748 t-0.0000035 t^{2}\right), \quad t \geq 0 .
$$

Finally, the economic agent preferences are described by CRRA utilities of the form (4) with risk aversion parameters $\gamma_{1}=0.5, \gamma_{2}=0.45, \gamma_{3}=0.4$ and $\beta_{1}=\beta_{2}=$ $\beta_{3}=0.5$.

In Fig. 1, we plot the value function $V(t, x)$ for a realization where $\tau_{1}=10$, $\tau_{2}=15$ and $\tau_{3} \geq 20$. The value function is obtained by solving three HJB equations of the form (6), restricting its solutions to the sets $\Delta_{i} \times \mathbb{R}^{+}, i \in\{1,2,3\}$, and finally, combining the corresponding graphs into a single one. Note the existence

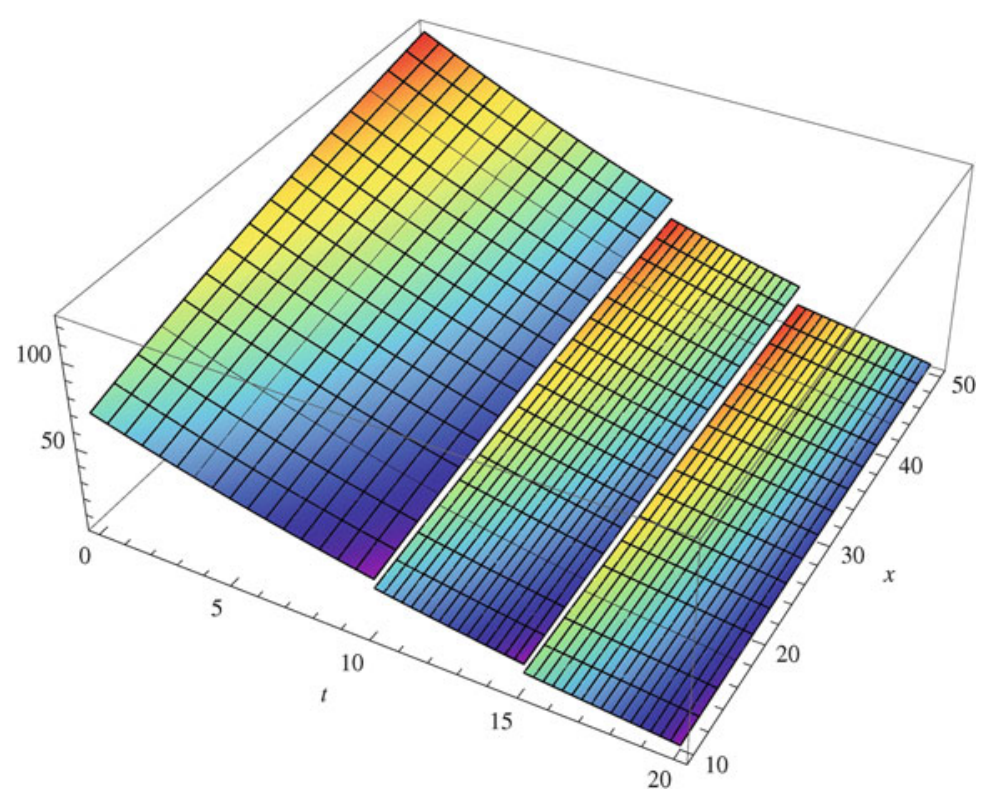

Fig. 1 Plot of the value function $V(t, x)$ for values $(t, x) \in[0,20] \times[10,50]$ and a realization where $\tau_{1}=10, \tau_{2}=15$ and $\tau_{3} \geq 20$ 


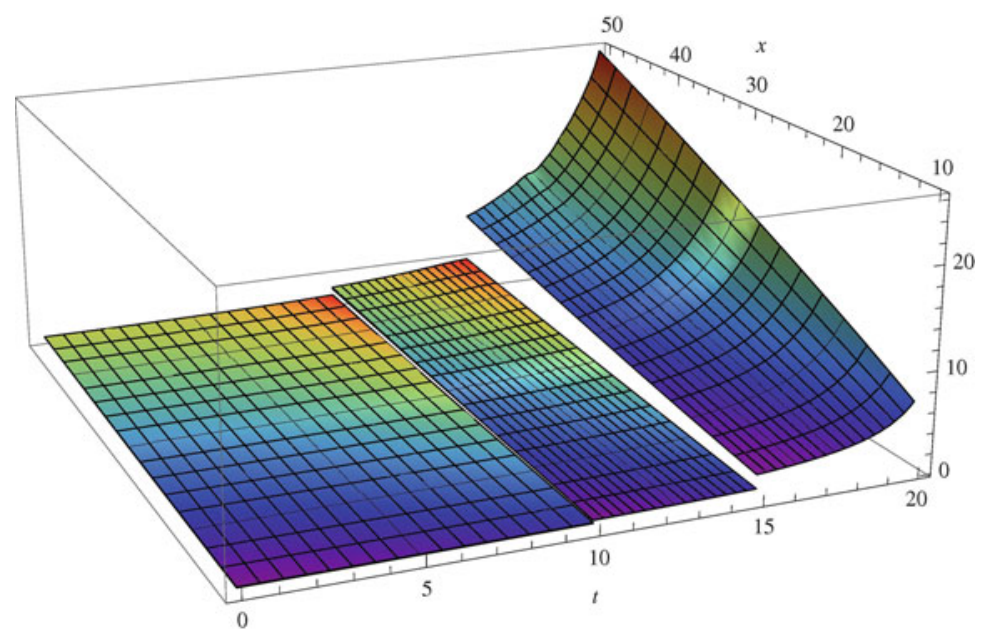

Fig. 2 Plot of the optimal consumptions $c^{*}(t, x)$ for values $(t, x) \in[0,20] \times[10,50]$ and a realization where $\tau_{1}=10, \tau_{2}=15$ and $\tau_{3} \geq 20$

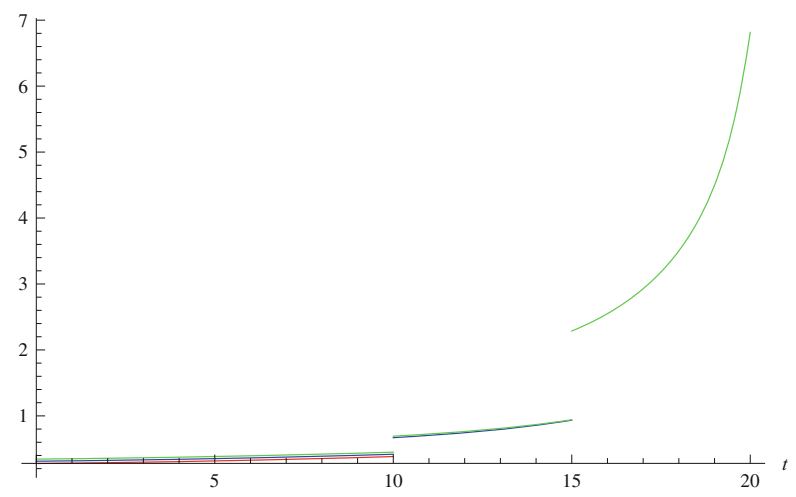

Fig. 3 Optimal consumptions $c^{*}$ for $x=10$

of discontinuities of the value function $V$ on the sets $t_{1}=10$ and $t_{2}=15$. Such behaviour is due to the strong dependence of the functional $J$ on the random variables $\tau_{1}, \tau_{2}, \tau_{3}$.

In Fig. 2, we plot the optimal consumptions $c_{i}^{*}(t, x), i \in\{1,2,3\}$, for a realization where $\tau_{1}=10, \tau_{2}=15$ and $\tau_{3} \geq 20$. The optimal consumptions $c_{i}^{*}(t, x)$ are obtained directly from the value function according to (5). Note that on the set $\Delta_{1} \times$ $\mathbb{R}^{+}$there exist three overlapping graphs, whereas on $\Delta_{2} \times \mathbb{R}^{+}$there are only two, and on $\Delta_{3} \times \mathbb{R}^{+}$only one. This is made clear in Figs. 3 and 4 , where a section of Fig. 2 is presented for a fixed value $x=10$. Note that the optimal consumptions are increasing functions of time $t$. Moreover, as should be expected, the optimal consumptions of the remaining goods jump up when one of the goods becomes 

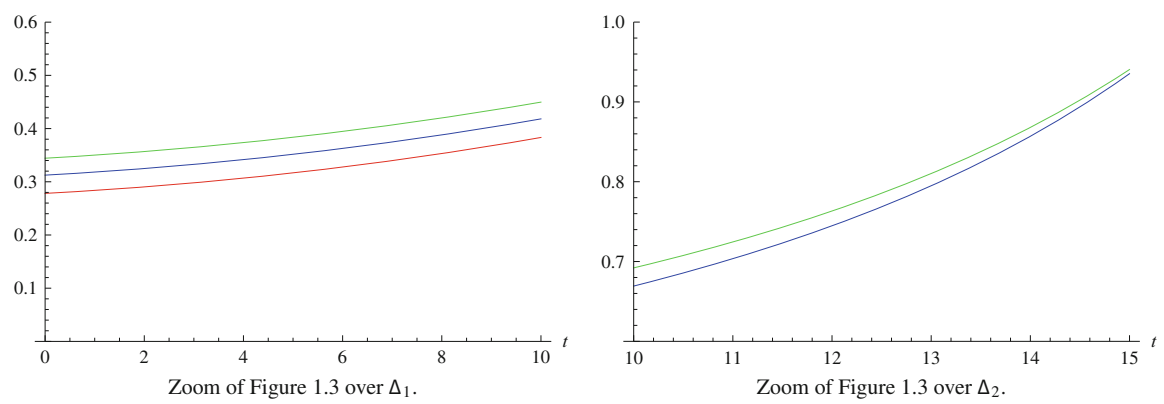

Fig. 4 A section of the optimal consumptions for $x=10$. The optimal consumption $c_{1}^{*}$ is plotted in red, while $c_{2}^{*}$ is plotted in blue and $c_{3}^{*}$ is plotted in green

unavailable, since the same amount of wealth can be distributed among a smaller number of goods.

\section{Conclusions}

We have studied a consumption-investment problem for an economic agent who is (i) consuming from a basket of $K$ goods that may become unavailable for consumption from some random time $\tau_{i}$ onwards, and (ii) investing her saving in a financial market consisting of one risk-free security and a fixed number of risky securities with diffusive terms driven by a standard multi-dimensional Brownian motion. We have applied the main abstract results of [19] to describe the value function associated with the stochastic optimal control problem under consideration here. We have then discussed the properties of the optimal strategies in the case where the economic agent has the same discounted CRRA utility functions for consumption and terminal wealth.

Acknowledgements A. A. Pinto thanks the financial support of LIAAD-INESC TEC through program PEst, USP-UP project, Faculty of Sciences, University of Porto, Calouste Gulbenkian Foundation, FEDER and COMPETE Programmes, and Fundação para a Ciência e a Tecnologia (FCT) through the Project "Dynamics and Applications" (PTDC/MAT/121107/2010). A. S. Mousa thanks Birzeit University and its Department of Mathematics.

\section{References}

1. Bellman, R.E.: On the theory of dynamic programming. Proc. Natl. Acad. Sci. USA 38, 716-719 (1952)

2. Bellman, R.E.: An introduction to the theory of dynamic programming. Rand Corporation Report pp. R-245 (1953) 
3. Bellman, R.E.: Dynamic programming and a new formalism in the calculus of variations. Proc. Natl. Acad. Sci. USA 40, 231-235 (1954)

4. Bellman, R.E.: Dynamic programming and stochastic control process. Inf. Control 1, 228-239 (1958)

5. Bismut, J.M.: Analyse convexe et probabilités, Thèse. PhD thesis, Faculté des Sciences de Paris, Paris (1973)

6. Bismut, J.M.: Théorie probabiliste du contrôle des diffusions. Mem. Am. Math. Soc. 4, 1-130 (1976)

7. Bismut, J.M.: An introductory approach to duality in optimal stochastic control. SIAM Rev. 20, 62-78 (1978)

8. Blanchet-Scalliet, C., El Karoui, N., Jeanblanc, M., Martellini, L.: Optimal investment decisions when time-horizon is uncertain. J. Math. Econ. 44(11), 1100-1113 (2008)

9. Boltyanski, V.G., Gamkrelidze, R.V., Pontryagin, L.S.: On the theory of optimal processes. Dokl. Akad. Nauk SSSR 10, 7-10 (1956)

10. Boltyanski, V.G., Gamkrelidze, R.V., Pontryagin, L.S.: The theory of optimal processes, I: the maximum principle. Am. Math. Soc. Transl. 2, 341-382 (1961)

11. Duarte, I., Pinheiro, D., Pinto, A.A., Pliska, S.R.: Optimal life insurance purchase, consumption and investment on a financial market with multi-dimensional diffusive terms. Optimization 63, $1737-1760$ (2014)

12. Fleming, W.H., Rishel, R.W.: Deterministic and Stochastic Optimal Control. Springer, New York (1975)

13. Fleming, W.H., Soner, H.M.: Controlled Markov Processes and Viscosity Solutions, 2nd edn. Springer, New York (2006)

14. Florentin, J.J.: Optimal control of continuous time, markov, stochastic systems. J. Electron. Control 10, 473-488 (1961)

15. Florentin, J.J.: Partial observability and optimal control. J. Electron. Control 13, 263-279 (1962)

16. Haussmann, U.G.: General necessary conditions for optimal control of stochastic systems. Math. Prog. Study 6, 34-48 (1976)

17. Karatzas, I., Shreve, S.: Methods of Mathematical Finance. Springer, New York (1998)

18. Kushner, H.J.: Optimal stochastic control. IRE Trans. Auto. Control AC-7, 120-122 (1962)

19. Mousa, A.S., Pinheiro, D., Pinto, A.A.: A family of stochastic optimal control problems with multiple random time horizons (2014, submitted)

20. Pliska, S.R., Ye, J.: Optimal life insurance purchase and consumption/investment under uncertain lifetime. J. Bank. Financ. 31, 1307-1319 (2007)

21. Yong, J., Zhou, X.Y.: Stochastic Controls: Hamiltonian Systems and HJB Equations. Springer, New York (1999) 